\title{
DECONVOLUTION OF GAMMA RAY SPECTRA USING SINGULAR VALUE DECOMPOSITION OF MATRICES
}

\author{
László HANKA \\ Óbuda University, Budapest, Hungary \\ hanka.laszlo@bgk.uni-obuda.hu
}

\begin{abstract}
The process of identification of radioactive isotopes using gamma ray spectrum produced by scintillation detectors is a fundamental problem in physics. Military applications also require fast and efficient methods, especially in field conditions, for identifying unknown isotopes. The fundamental problem is the relationship between the observed gamma ray spectrum given by the detector and the real spectrum. This problem can be treated as a mathematical problem. The relationship between the real and the observed spectrum can be described by a linear algebraic equation system. In the previous article Cholesky-decomposition has been applied. In this article one more independent mathematical tool is proposed to solve the linear system efficiently.
\end{abstract}

KEYWORDS: gamma ray spectrum, deconvolution, singular value decomposition, active set method, Matlab

\section{Introduction}

Figure no.1 shows a typical and most general gamma-ray spectrum of a monoenergetic gamma ray source.

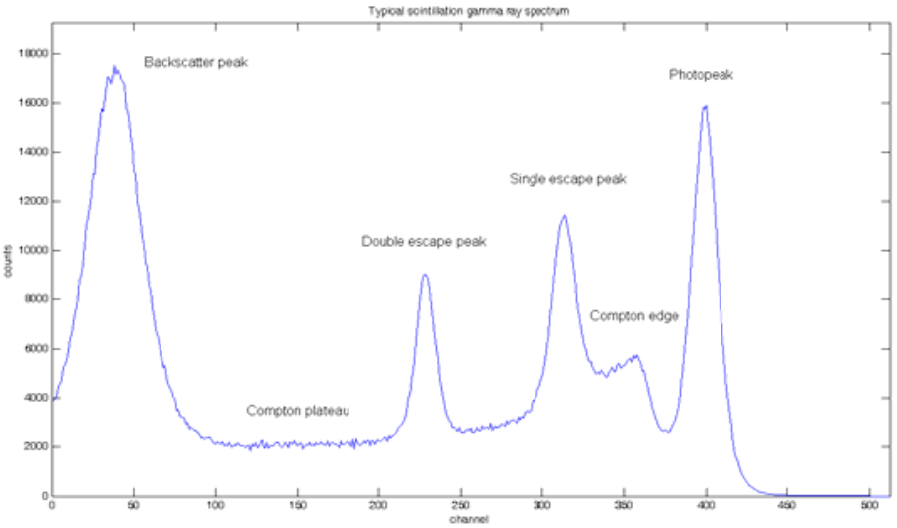

Figure no. 1: Typical gamma spectrum of a monoenergetic gamma source (Source: Author) 
This spectrum is the response of the detector for single gamma energy, when the real spectrum can be described by a single Dirac-delta function (Berger \& Seltzer, 1972; Meng \& Ramsden, 2000; Morhac, 2006). According to the statistical property of the radioactive decay and the background radiation, and due to the statistical feature of the fluctuations and the noise of electronic components, the statistical affects of absorption, scattering, backscattering, and so on, the "peaks" are not exact delta functions, namely not only one channel contains large number of counts, but peaks are widened. These peaks can be described approximately by a Gaussian distribution function. This is true not only for the photopeak but for the backscatter peak and for "escape peaks" as well. This feature is just the same for the Compton-edge (Gerasimov, 1995; Gopinath \& Gopala, 1999; Los Arcos, 1996). It is not a "sharp edge", due to the statistical fluctuations it becomes "round", the edge is broadening, the transition from the Compton plateau to the photopeak is smooth.

This observed spectrum mathematically a vector, denoted by $\mathbf{y}$. Components of $\mathbf{y}$ are observed number of counts in channels of analyser (Bouchet, 1995; Morhac, 2006). The real spectrum is vector $\mathbf{x}$, and the relationship between $\mathbf{x}$ and $\mathbf{y}$ is described by a linear system

$$
\mathbf{R x}=\mathbf{y}
$$

where $\mathbf{R}$ is the response matrix of the detector. The response matrix must be constructed for every specific detector. Deconvolution is the procedure by which the real spectrum $\mathbf{x}$ is determined using the response matrix $\mathbf{R}$ and the observed spectrum y (Meng \& Ramsden, 2000; Morhac, 2006).

In this study an efficient procedure is proposed for deconvolution, using the fundamental notion of linear algebra, that is called Singular Value Decomposition of a matrix.

\section{Deconvolution as an Optimization Problem}

The deconvolution problem, in other words the problem of solving the linear system

$$
\mathbf{R x}=\mathbf{y} ;
$$

can be considered as an optimization problem (Housholder, 1964; Stoer \& Burlish, 1991). Since the system is practically unsolvable, the problem instead is finding the best approximate solution of the system, so the following optimization problem must be solved:

$$
\Phi(\mathbf{x})=\|\mathbf{R} \mathbf{x}-\mathbf{y}\|^{2} \rightarrow \min
$$

provided that $\mathbf{x} \geq \mathbf{0}$ (a feasible solution must be positive semidefinite), since every coordinate of a solution is a number of counts in one channel, which can't be negative.

It must be emphasized, that

$$
\boldsymbol{\varepsilon}=\mathbf{R x}-\mathbf{y}
$$

is the error of a solution, so the sum of squares of errors must be minimized. According to the definition of Euclidean norm (Bellmann, 1960; Strang, 2003), this function can be written in the form

$$
\begin{aligned}
\Phi(\mathbf{x}) & =\|\mathbf{R x}-\mathbf{y}\|^{2}=(\mathbf{R x}-\mathbf{y})^{T}(\mathbf{R x}-\mathbf{y})= \\
& =\mathbf{x}^{T} \mathbf{R}^{T} \mathbf{R} \mathbf{x}-2 \mathbf{x}^{T} \mathbf{R}^{T} \mathbf{y}+\mathbf{y}^{T} \mathbf{y}
\end{aligned}
$$

The least square estimate of $\mathbf{x}$ is found by setting the derivative of $\Phi(\mathbf{x})$ equal to zero:

$$
\Phi^{\prime}(\mathbf{x})=2 \mathbf{R}^{T} \mathbf{R} \mathbf{x}-2 \mathbf{R}^{T} \mathbf{y}=0
$$

After dividing by 2 we get the well known Gaussian equation

$$
\mathbf{R}^{T} \mathbf{R x}=\mathbf{R}^{T} \mathbf{y}
$$

In this study, instead of solving the Gaussian equation, the optimization problem itself will be studied.

To obtain a smoother, positive semidefinite solution, one more condition is 
required: together the sum of squares of errors the sum of squares of elements of $\mathbf{x}$ vector is also minimized. Taking into consideration of this requirement, the following optimization problem must be solved:

$$
\Phi(\mathbf{x})=\frac{1}{2}\|\mathbf{R x}-\mathbf{y}\|^{2}+\frac{\alpha}{2}\|\mathbf{x}\|^{2} \rightarrow \min
$$

where $\alpha$ is the regularization parameter. The one half factor is applied for convenience. For differentiating the function, performing operations is recommended:

$\Phi(\mathbf{x})=\frac{1}{2} \mathbf{x}^{T} \mathbf{R}^{T} \mathbf{R} \mathbf{x}-\mathbf{x}^{T} \mathbf{R}^{T} \mathbf{y}+\frac{1}{2} \mathbf{y}^{T} \mathbf{y}+\frac{\alpha}{2} \mathbf{x}^{T} \mathbf{x} ;(9)$

Taking the derivative of this function the following necessary condition is obtained

$$
\Phi^{\prime}(\mathbf{x})=\mathbf{R}^{T} \mathbf{R} \mathbf{x}-\mathbf{R}^{T} \mathbf{y}+\alpha \mathbf{x}=0 .
$$

which is equivalent to the following, generalized Gaussian equation:

$$
\left(\mathbf{R}^{T} \mathbf{R}+\alpha\right) \mathbf{x}=\mathbf{R}^{T} \mathbf{y}
$$

So it is proved, that the optimization problem is equivalent to the original deconvolution problem, using the idea of regularization of coefficient matrix. In this study an efficient procedure is proposed for solving the optimization of the function

$$
\Phi(\mathbf{x})=\frac{1}{2}\|\mathbf{R x}-\mathbf{y}\|^{2}+\frac{\alpha}{2}\|\mathbf{x}\|^{2} ; \mathbf{x} \geq \mathbf{0}
$$

where $\mathbf{R}$ is the response matrix of the detector, and $\mathbf{y}$ is the observed spectrum.

\section{Singular Value Decomposition}

The Singular Value Decomposition (SVD) of a matrix is one of the best factorizations of any matrix (Strang, 2003; Bellmann, 1960). The advantage of this factorization on the one hand that it can be applied for any matrix. The matrix doesn't need to be neither nonsingular nor quadratic. The SVD of the $n \times k$ matrix $\mathbf{A}$ is the following product:

$$
\mathbf{A}=\mathbf{U S V}^{\mathbf{T}}
$$

where $\mathbf{U}$ is a quadratic orthogonal $n \times n$ matrix, $\mathbf{V}$ is also a quadratic orthogonal $k \times k$ matrix, the superscript " $T$ " refers to the transpose of matrix $\mathbf{V}$. Due to the fact, that $\mathbf{U}$ and $\mathbf{V}$ are orthogonal matrices, the inverse of these matrices can be given without any calculations, since the inverse of an orthogonal matrix is simply it's transpose. So giving the inverse of an orthogonal matrix, only the position of entries must be modified. Therefore

$$
\mathbf{U}^{-1}=\mathbf{U}^{\mathrm{T}} ; \quad \mathbf{V}^{-1}=\mathbf{V}^{\mathrm{T}}
$$

Finally $\mathbf{S}$ is a general diagonal $n \times k$ matrix, of the following form:

$$
\mathbf{S}=\left[\begin{array}{cccccc}
\sigma_{1} & 0 & \ldots & 0 & \ldots & 0 \\
0 & \sigma_{2} & \ldots & 0 & \ldots & 0 \\
\ldots & \ldots & \ldots & \ldots & \ldots & \ldots \\
0 & 0 & \ldots & \sigma_{r} & \ldots & 0 \\
\ldots & \ldots & \ldots & \ldots & \ldots & \ldots \\
0 & 0 & \ldots & 0 & \ldots & 0
\end{array}\right] \in \mathbb{R}^{n \times k}
$$

As it can be seen, in matrix $\mathbf{S}$ nonzero entries can only be found in the main diagonal. The first $r$ nonzero entries in the main diagonal are the so called singular values, by definition in descending order, where $r$ is the rank of matrix $\mathbf{A}$, and every other entry in $\mathbf{S}$ is zero.

If matrix $\mathbf{A}$ is a quadratic $n \times n$ matrix, like in the problem that is being studied in this article, naturally every matrix, $\mathbf{U}, \mathbf{S}$ and $\mathbf{V}$ are quadratic $n \times n$ matrices.

One of the most important applications of SVD is solving linear algebraic systems, like

$$
\mathbf{A x}=\mathbf{y}
$$

when the coefficient matrix A has "bad properties", for example if it's determinant is close to zero or if condition number is great.

Recalling the most important equation from the previous section 


$$
\left(\mathbf{R}^{T} \mathbf{R}+\alpha\right) \mathbf{x}=\mathbf{R}^{T} \mathbf{y}
$$

and introducing simpler notations

$$
\mathbf{A}=\mathbf{R}^{T} \mathbf{R}+\alpha ; \quad \mathbf{y}=\mathbf{R}^{T} \mathbf{y} ;
$$

(the second simplification doesn't cause confusion), it is clear that the necessary condition of the optimization problem is truly equivalent to the system

$$
\mathbf{A x}=\mathbf{y} .
$$

For solving this system the inverse of matrix $\mathbf{A}$ is required, but if one of the previous properties hold, the inverse matrix can't be given accurately (Hanka, 2020) since calculations are extremely sensitive to error. Applying Matlab an error message is obtained like the following:

"Matrix is close to singular or badly scaled. Results may be inaccurate. $\mathrm{RCOND}=3.526328 \mathrm{e}-44 . "$

In this study, the response matrix of the detector (Berger \& Seltzer, 1972; Morhac, 2006) has extremely big condition number (Hanka, 2020; Stoer \& Burlish, 1991), therefore the inverse of $\mathbf{A}$ is not accurate for further calculations. But SVD of $\mathbf{A}$ helps solving this problem.

On the other hand, the advantage of SVD is that a generalized inverse, the Moore-Penrose inverse of any matrix can be given using this factorization.

The Moore-Penrose inverse of $\mathbf{A}$ is the following product (Strang, 2003):

$$
\mathbf{A}^{+}=\mathbf{V S}^{+} \mathbf{U}^{\mathbf{T}}
$$

For the general inverse instead of " -1 " the "+" superscript is used in the notation. The first and third factor in this product is clear, and the second factor is obviously the general inverse of matrix $\mathbf{S}$. The form of $\mathbf{S}^{+}$is as follows:

$$
\mathbf{S}^{+}=\left[\begin{array}{cccccc}
\frac{1}{\sigma_{1}} & 0 & \ldots & 0 & \ldots & 0 \\
0 & \frac{1}{\sigma_{2}} & \ldots & 0 & \ldots & 0 \\
\ldots & \ldots & \ldots & \ldots & \ldots & \ldots \\
0 & 0 & \ldots & \frac{1}{\sigma_{r}} & \ldots & 0 \\
\ldots & \ldots & \ldots & \ldots & \ldots & \ldots \\
0 & 0 & \ldots & 0 & \ldots & 0
\end{array}\right] \in \mathbb{R}^{k \times n}(21)
$$

If $\mathbf{S}$ in general is a $n \times k$ matrix, the inverse, $\mathbf{S}^{+}$is $k \times n$ matrix. Applying SVD of $\mathbf{A}$, the system is replaced by the following:

$$
\mathbf{U S V}^{\mathbf{T}} \mathbf{x}=\mathbf{y}
$$

This system can be solved in three steps:

$$
\begin{aligned}
& \mathbf{S V}^{\mathbf{T}} \mathbf{x}=\mathbf{U}^{\mathbf{T}} \mathbf{y} \\
& \mathbf{V}^{\mathbf{T}} \mathbf{x}=\mathbf{S}^{+} \mathbf{U}^{\mathrm{T}} \mathbf{y} \\
& \mathbf{x}=\mathbf{V} \mathbf{S}^{+} \mathbf{U}^{\mathbf{T}} \mathbf{y}
\end{aligned}
$$

As it can be seen in the last row, after all the Moore-Penrose inverse is applied. The advantage of this procedure is clear in light of the above. In Moore-Penrose inverse every factor can be given easily.

It must be underlined, that this general inverse is not an inverse in classic sense, the product $\mathbf{A A}^{+}$is not equal to the indentity matrix. Instead the general inverse satisfy the Moore-Penrose conditions:

$$
\mathbf{A} \mathbf{A}^{+} \mathbf{A}=\mathbf{A} ; \quad \mathbf{A}^{+} \mathbf{A} \mathbf{A}^{+}=\mathbf{A}^{+}
$$

The only remaining problem regarding SVD, that how it can be constructed. In our study for every calculation Matlab has been used. In Matlab there is a built in procedure for producing $\mathbf{U}, \mathbf{S}$ and $\mathbf{V}$. The appropriate command is the following:

$$
\text { “[U,S, V }]=\operatorname{svd}(\mathbf{A}) " .
$$


This procedure requires lots of calculations, for a $n \times n$ matrix the number of necessary calculations is proportional to $n^{3}$, but applying an average performance computer, for a $512 \times 512$ matrix, like the response matrix of the detector, that is being studied, it takes only a fraction of a second.

Testing the efficiency of SVD, first of all an artificial gamma spectrum was constructed, that consist of six pure Gaussian curves. One pure Gaussian curve can be considered as a response to one monoenergetic gamma source. Figure no. 2 illustrates the six Gaussians.

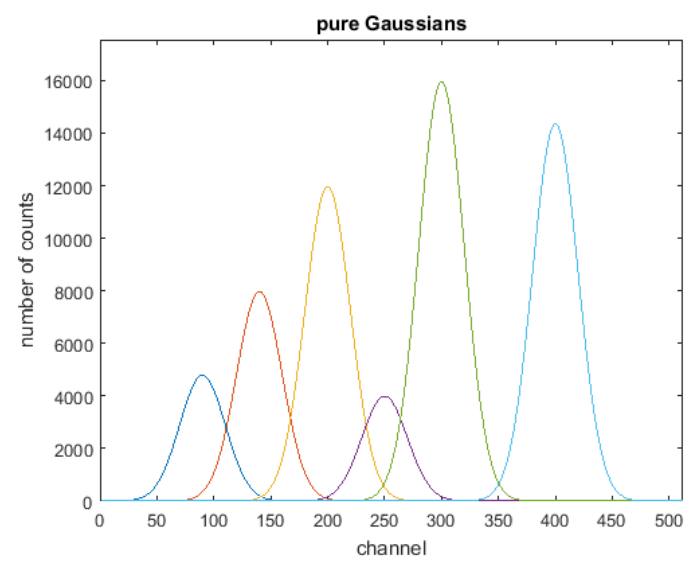

Figure no. 2: Pure Gaussian curves

(Source: Author)

The sum of these curves (Figure no. 3) can be considered as an artificial gamma pectrum of the source emitting 6 gamma energies.

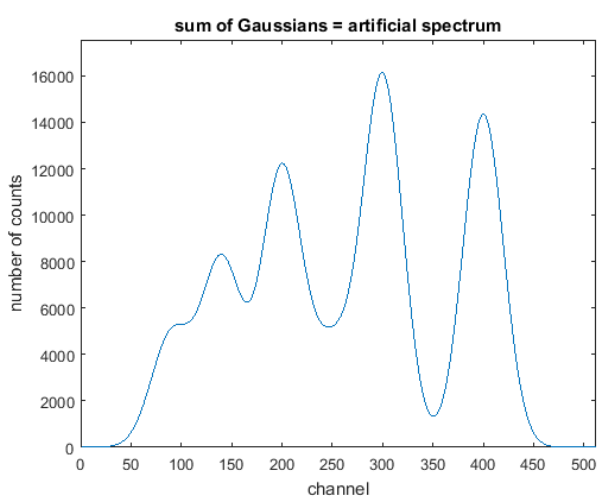

Figure no. 3: Artificial spectrum

(Source: Author)
This artificial spectrum consists of overlapping Gaussian curves, and there is one hidden curve. Applying the SVD for this artificial spectrum, the deconvolution procedure produces the six gamma lines exactly (Figure no. 4).

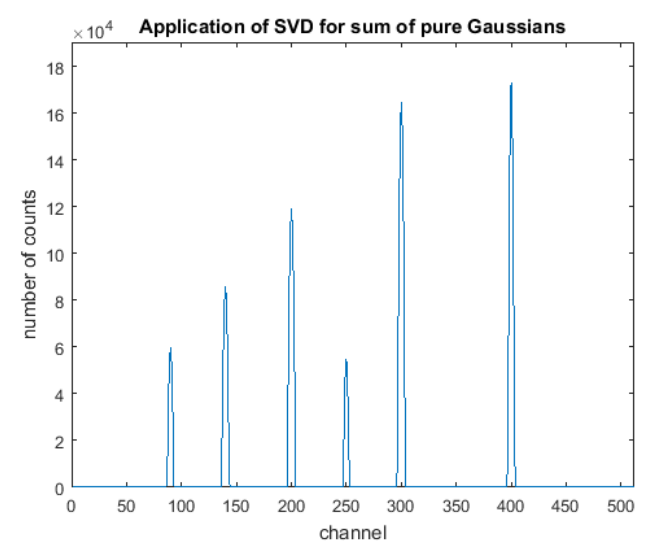

Figure no. 4: Deconvolution of artificial spectrum

(Source: Author)

As it is clear, the overlapping curves are separated and the hidden line also can be seen separately.

For further study the artificial spectrum is modified by some normally distributed noise (Figure no. 5).

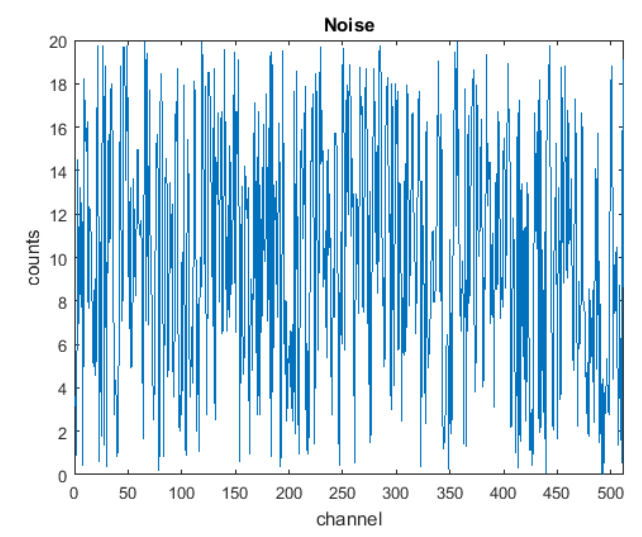

Figure no. 5: Normally distributed noise (Source: Author)

This noise was added to the pure artificial spectrum, and for the noisy spectrum the same procedure, the SVD has been applied. The result of the procedure can be seen on Figure no. 6 . 


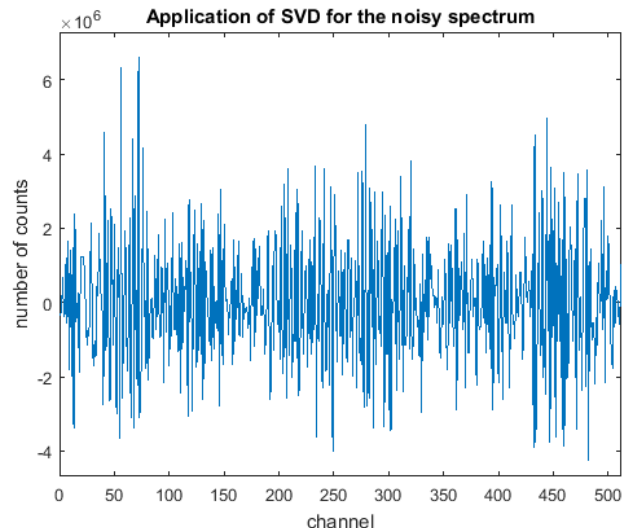

Figure no. 6: Deconvolution of noisy spectrum

(Source: Author)

Comparing Figure no. 4 and Figure no. 6 it is clear that the solution obtained makes no sense. It is strongly oscillating, and has lots of negative coordinates, so it is absolutely not feasible. We can conclude that SVD is very sensitive to noise, in other words to the error, so unfortunately it doesn't work.

Consequently, the direct application of SVD doesn't solve the problem. But, as it will be illustrated in the following section, a proper extension of SVD can be very efficient procedure. The proposed extension is the active set method, which will be discussed below.

\section{The Active Set Method}

In order to define and apply the active set method (Landi \& Zama, 2006), some notations must be introduced. Let $\Omega$ be the set of feasible points in $\mathbf{R}^{n}$ (the nonnegative orthant):

$$
\Omega=\left\{\mathbf{x}=\left[x_{1}, x_{2}, \ldots, x_{n}\right] \in \mathbf{R}^{n} \mid x_{i} \geq 0 ; I=1,2, \ldots, n\right\}
$$

Furthermore let $\mathfrak{I}$ be the set of indices: $\mathfrak{I}=\{1,2, \ldots, n\}$. The notion of "active set" is defined in the following way: for particular index $i \in \mathfrak{I}$ the $x_{i} \geq 0$ condition is said to be active if $x_{i}=0$. Consequently the active set $\Theta$, which is a subset of the set of indices, is defined by the following way:

$$
\Theta=\left\{i \in \mathfrak{I} \mid x_{i}=0\right\} \subset \mathfrak{J}
$$

Simply to say, the active set is the set of subscripts of zero coordinates in a solution.

For deconvolution the Singular Value Decomposition method is combined with an iterative procedure. For justifying the necessity of iteration, properties of the solution to the system must be studied. Since the system $\mathbf{A x}=\mathbf{y}$ can' be solved exactly, the solution $\mathbf{x}$ is never accurate. The deviation between the accurate solution and the obtained solution can be given by $\delta \mathbf{x}$. Using this notion $\mathbf{x}+\delta \mathbf{x}$ satisfies the system exactly:

$$
\mathbf{A}(\mathbf{x}+\delta \mathbf{x})=\mathbf{y}
$$

So, if solution $\mathbf{x}$ is known, only the deviance vector $\delta \mathbf{x}$ must be determined for a more accurate solution. Rearranging the previous system, it is clear that the deviance vector is the solution to the system below:

$$
\mathbf{A} \delta \mathbf{x}=\mathbf{y}-\mathbf{A} \mathbf{x}=\mathbf{r} .
$$

where new notation $\mathbf{r}$ is introduced. Now the structure of the iteration is clear. Assume that in the $k$ th step the solution is known $\mathbf{x}^{(k)}$. Using this vector, the deviance vector $\delta \mathbf{x}^{(k)}$ is determined by solving the system

$$
\mathbf{A} \delta \mathbf{x}=\mathbf{y}-\mathbf{A} \mathbf{x}^{(k)}=\mathbf{r}^{(k)}
$$

and in the $(k+1)$ th step the approximate solution is the vector

$$
\mathbf{x}^{(k+1)}=\mathbf{x}^{(k)}+\delta \mathbf{x}^{(k)} .
$$

As it is illustrated on Figure no. 6. application of SVD may produce negative solutions. Avoiding negative coordinates, two possibilities are illustrated here. Temporarily using different notation for the solution in the $(k+1)$ th step, 


$$
\mathbf{x}^{(k+1)}=\mathbf{x}^{(k)}+\delta \mathbf{x}^{(k)}
$$

on the one hand the new solution can be defined by a simple projection onto the set of feasible solutions $\Omega$ in the following way:

$$
x_{i}^{(k+1)}=\left\{\begin{array}{l}
0 ; \text { if } x_{i}^{\prime(k+1)} \leq 0 \\
x_{i}^{\prime}{ }_{i}^{(k+1)}, \quad \text { else }
\end{array} ; i \in \mathfrak{I}\right.
$$

Simply to say, negative coordinates are substituted by 0 in a new solution.

On the other hand a more precise procedure can be the following. The new solution is defined by the formula

$$
\mathbf{x}^{(k+1)}=\mathbf{x}^{(k)}+\gamma_{k}\left(\mathbf{x}^{\prime^{(k+1)}}-\mathbf{x}^{(k)}\right)
$$

where $\left.\left.\gamma_{k} \in\right] 0,1\right]$ is the greatest constant for which $\mathbf{x}^{(k+1)} \geq \mathbf{0}$. In this study the latter procedure has been used.

This is the point where the active set method is applied. A basic requirement obtaining only feasible solutions in every iteration step, and furthermore it must be taken into account, that an optimization problem must be solved.

Selecting any subscript $i$ from the active set $\Theta$, where $x_{i}$ coordinate is zero, the modification of the coordinate $\delta x_{i}^{(k)}$ must be examined. Only feasible solutions are acceptable, consequently for this subscript only nonnegative solution of the system

$$
\mathbf{A} \delta \mathbf{x}=\mathbf{r}^{(k)}
$$

is accepted. Ensuring this requirement on the one hand a matrix $\mathbf{B}^{(k)}$ is constructed from the identity matrix: if $i \in \mathfrak{I}$ then the entry $e_{i i}=1$ is replaced by 0 , and instead of solving the previous system, the following system will be solved for vector $\mathbf{z}$ :

$$
\mathbf{A B} \mathbf{B}^{(k)} \mathbf{z}=\mathbf{r}^{(k)}
$$

Using SVD in every iteration step, the solution of this system $\mathbf{z}^{(k)}$ is produced. After all the new solution is the vector

$$
\mathbf{x}^{\prime(k+1)}=\mathbf{x}^{(k)}+\mathbf{B}^{(k)} \mathbf{z}^{(k)} ;
$$

and the new approximation is

$$
\mathbf{x}^{(k+1)}=\mathbf{x}^{(k)}+\gamma_{k}\left(\mathbf{x}^{\prime^{(k+1)}}-\mathbf{x}^{(k)}\right) ;
$$

as it was discussed earlier.

On the other hand, taking into account the active set, one more idea is applied. Since an optimization problem is solved at the same time, the partial derivative

$$
\frac{\partial \Phi}{\partial x_{i}^{(k)}}
$$

is calculated for every $i \in \Theta$. The greatest positive derivatives are selected, and the corresponding subscripts are deleted from the active set, since if these derivatives are positive, it means that modifying the zero coordinates by a positive quantity, the solution is getting closer and closer to the optimal solution.

Choosing a small $\varepsilon>0$, the stopping criterion of the iteration can be

$$
\left\|\mathbf{A} \mathbf{x}^{(k+1)}-\mathbf{y}\right\|<\varepsilon .
$$

or a specific number of steps can be specified (Stoer \& Burlish, 1991).

Obviously the active set varies in every iteration step, since zero coordinates belong to different subscripts in the new solution, and due to the operation with partial derivatives. This is why the set is called "active".

The disadvantage of this procedure, that matrix $\mathbf{A} \mathbf{B}^{(k)}$ is different in each step due to 2activity" of the set $\Theta$, so the Singular Value Decomposition of this coefficient matrix also must be performed in every step, so the algorithm requires lost of operations. But, applying this procedure for a real spectrum, using a $512 \times 512$ response matrix, the complete calculation takes only a couple of seconds. 
The result of the application of the proposed procedure for noisy spectrum can be seen on Figure no. 7.
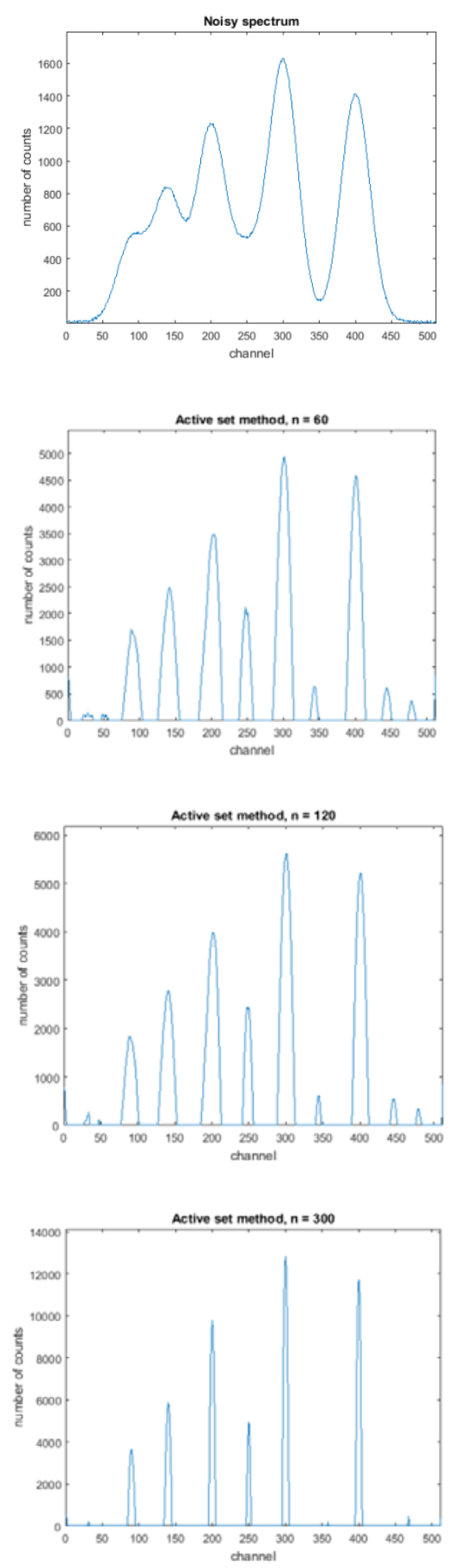

Figure no. 7: Deconvolution of noisy spectrum using SVd and active set method, $n$ is the number of iterations

(Source: Author)
It can be seen on Figure no. 7 how the approximate solution is getting more and more accurate. The overlapping Gaussians are separated, fake peaks gradually disappear and the photopeaks are becoming more and more similar to Dirac delta functions. Fitting Gaussian curves to these photopeaks, the mean values are exactly equal to the original positions of artificial gamma energies. Calculations took only less than 5 seconds.

So it seems, that the proposed procedure works, at least for artificial spectrum. In the following section it is proved, that this algorithm can also be applied for a real spectrum as well.

\section{Application for Real Spectrum}

For testing the proposed algorithm for real problem, the mixed spectrum of nuclides Co-60, Ba-133 and $\mathrm{Cs}-137$ was chosen. The spectrum of this mixture can be seen on Figure no. 8.

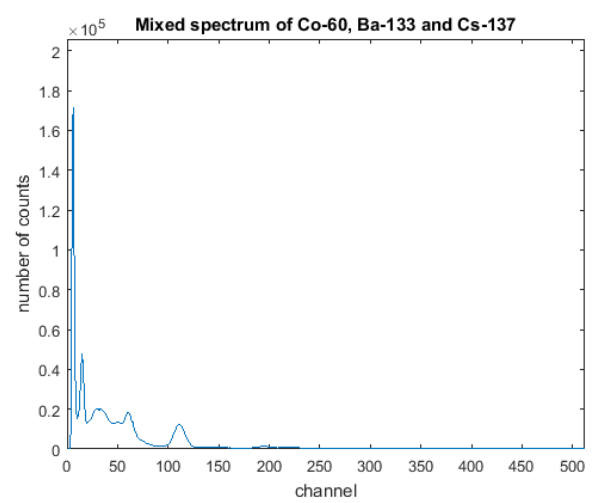

Figure no. 8: Mixed spectrum of nuclides Co-60, $\mathrm{Ba}-133$ and $\mathrm{Cs}-137$

(Source: Author)

The algorithm, described in sections 2-4 has been applied for this spectrum. The entire algorithm is exactly the same. The only difference is that in this real case the response matrix $\mathbf{R}$ of the detector is different (Berger \& Seltzer, 197; Morhac, 2006), since for real spectra a completely different specific response matrix must be constructed. 
On Figure no. 9 the result of the procedure can be seen. It took only less than 5 seconds again, so the necessary time is negligible. As it is clear on the graph, 5 intensive, sharp Dirac delta functions can be seen. These are the characteristic gamma lines of nuclides in the mixture.

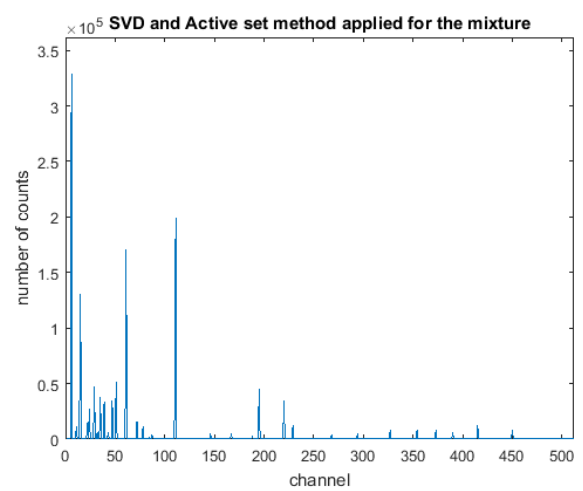

Figure no. 9: Deconvolution of mixed spectrum of nuclides Co-60, Ba-133 and Cs-137, using SVD and active set method (Source: Author)

The exact position of these peaks can be identified by fitting Gaussian curve for every peak.

Performing the necessary calculations the following is obtained:
The exact energy of gamma lines of Co-60 is $\mathrm{E}_{1}=1,1732 \mathrm{MeV}$ and $\mathrm{E}_{2}=1,3325 \mathrm{MeV}$. The proposed algorithm produces $\mathrm{E}_{1}=1,1727 \mathrm{MeV}$ and $\mathrm{E}_{2}=1,3256 \mathrm{MeV}$.

The exact energy of gamma lines of Ba-133 is $\mathrm{E}_{1}=0,0809 \mathrm{MeV}$ and $\mathrm{E}_{2}=0,3560 \mathrm{MeV}$. The proposed algorithm produces $\mathrm{E}_{1}=0,0811 \mathrm{MeV}$ and $\mathrm{E}_{2}=0,3585 \mathrm{MeV}$.

The exact energy of gamma line of $\mathrm{Cs}-137$ is $\mathrm{E}=0,6616 \mathrm{MeV}$. The proposed algorithm produces $\mathrm{E}=0,6613 \mathrm{MeV}$.

We proved, that the proposed algorithm works even in a real case.

\section{Conclusions}

In this study an efficient deconvolution algorithm is proposed for identifying gamma sources. As it was proved in section 4 and 5, that the Singular value Decomposition of matrices, extended by the active set method proved to be efficient tool for solving the ill-posed linear system, which is fundamental in gamma spectroscopy. For these calculations Matlab software is recommended due to built in functions. In this study every calculation and every Figure has been made using Matlab.

\section{REFERENCES}

Bellmann, R. (1960). Introduction to Matrix Analysis. New York: McGraw-Hill Book Company.

Berger, M. J., \& Seltzer, S. M. (1972). Response function for sodium iodide scintillation detectors. Nuclear Instrumens and Methods, Vol. 104, 317-332.

Bouchet, L. (1995). A comparative study of deconvolution methods of gamma-ray spectra. Astronomy and Astrophysics Supplement Series, Ser.113, 167-183.

Gerasimov, S. A. (1995). A direct method for separating peak multiplet in X-ray and gamma-ray spectra. Applied radiation and isotopes, Vol. 46, 181-184.

Gopinath, D. V., \& Gopala, K. (1999). Analitical computation of the Compton continuum in gamma-ray spectrometry. Radiation Physics and Chemistry, Vol. 56, Issue 5-6, 525-534.

Halmos, P. R (1958). Finite-dimensional vector spaces. Princeton, NJ: Van Nostrand.

Hanka, L. (2020). Identification of radioactive isotopes using Cholesky-decomposition of matrices. Revista Academiei Forțelor Terestre / Land Forces Academy Review, Vol. 97, Issue 1, 76-84. Paper: 10.2478/raft-2020-0010. 
Householder, A. S. (1964). The Theory of Matrices in Numerical Analysis. New York: Blaisdell.

Jandel, M., Morhac, M., Kliman, J., Krupa, L., Matousek, V., Hamilton, J. H., \& Ramaya, A. V. (2004). Decomposition of continuum $\gamma$-ray spectra using syhthesized response matrix. Nuclear Instruments \& Methods in Phisics Research, A 516, 172-183.

Landi, G., \& Zama, F. (2006). The active set method for nonnegative regularization of ill-posed problem. Applied Mathematics and Computation, Vol. 175, 715-729.

Los Arcos, J. M. (1996). Gamma-ray spectra deconvolution by maximum-entropy methods. Nuclear Instruments and Methods in Physics Research, Section A, Vol. 369, Issue 2-3, 634-636

Meng, L. J., \& Ramsden D. (2000). An Inter-comparison of Three SpectralDeconvolution Algorithms for Gamma-ray Spectroscopy. IEEE Transactions on Nuclear Science, Vol. 47. No. 4.

Morhac, M. (2006). Deconvolution methods and their applications in the analysis of gamma-ray spectra. Nuclear Instruments and Methods in Physics Research, Section A, Vol. 559, 119-123.

Shi, H.-X., Chen, B.-X., Li, T.-Z., \& Yun, D. (2002). Precise Monte Carlo simulation of gamma ray response function for an $\mathrm{NaI}(\mathrm{Tl})$ detector. Applied Radiation and Isotopes, Vol. 57, Issue 4, 517-524.

Stoer, J., \& Burlish, R. (1991). Introduction to numerical analysis. New-York: Spinger. Strang, G. (2003). Introduction to Linear Algebra. MIT: Wellesley-Cambridge Press. 